\title{
Reappraisal of Spontaneous Closure Rate of Idiopathic Full-Thickness Macular Holes
}

\author{
Atsushi Sugiyama, Mitsuhiro Imasawa", Tatsuya Chiba and Hiroyuki Iijima
}

Department of Ophthalmology, Faculty of Medicine, University of Yamanashi, Shimokato 1110, Chuo, Yamanashi, 4093898, Japan

\begin{abstract}
We retrospectively reviewed the records of 142 eyes of 138 patients with idiopathic full-thickness macular hole. Spontaneous closure of idiopathic full-thickness macular hole was observed in five eyes (3.5\%) of four patients before the planned vitrectomy. In the era when surgical treatment was not available for macular hole, the rate of spontaneous closure of idiopathic full-thickness macular hole was reported as $6.2 \%$. Among several case reports on spontaneous closure of idiopathic full-thickness macular hole based on the optical coherence tomography images only one study reported the rate of spontaneous closure as $2.7 \%$. According to the previous reports and our results, small idiopathic full-thickness macular holes may close spontaneously in a few percent of all macular hole cases. The rate of spontaneous closure may be affected by the waiting time before vitrectomy.
\end{abstract}

Keywords: Full-thickness macular hole, spontaneous closure, optical coherence tomography.

\section{TO THE EDITOR,}

During the era in which surgical treatment was not available for macular hole, Yuzawa et al. reported that spontaneous closure of macular hole was observed in six $(6.2 \%)$ of 97 patients with idiopathic full-thickness macular hole (IFTMH) [1]. Because the diagnosis of macular hole and its closure has been more accurately determined with the advent of optical coherence tomography (OCT), and because the macular hole surgery has become more popular and prevalent than before, we reappraised the rate of spontaneous closure of IFTMH in a retrospective study.

We retrospectively reviewed the records of 142 eyes of 138 patients with IFTMH who were referred to the University of Yamanashi Hospital between January 2005 and November 2009. Exclusion criteria were high myopia $<$ -8 diopters; short follow-up periods $<3$ months. The study population included 56 men and 82 women with mean age of 67.4 years (range: 50 to 83 years). IFTMH was diagnosed using OCT (Stratus OCT; Carl Zeiss Meditec, Dublin, California, USA). The 142 eyes were classified according to the OCT-based macular hole stage [2]: stage 2 (the posterior hyaloid is still attached to the hole edge in the OCT image), 45 eyes $(31.7 \%$ ); stage 3 (the posterior hyaloid is detached from the hole edge but not from the optic disk), 71 eyes (50\%); and stage 4 (complete posterior vitreous detachment), 26 eyes $(18.3 \%)$. Macular hole closure was defined as the presence of an uninterrupted sensory retina covering the fovea. They included eyes exhibiting residual subretinal space beneath the continuous sensory retina in the macula in the OCT image. Vitrectomy was planned in all eyes between 11 days and 154 days (mean: 65.2 days) after the initial

*Address correspondence to this author at the Department of Ophthalmology, Faculty of Medicine, University of Yamanashi, Shimokato 1110, Chuo, Yamanashi, 409-3898, Japan; Tel: +81 552739657;

Fax: +81 55273 6757; E-mail: mimasawa@yamanashi.acjp presentation in accordance with the recommended operation period within 6 months of onset [3].

Spontaneous closure of IFTMH was observed in five eyes $(3.5 \%)$ in four patients prior to the planned vitrectomy (Table 1). In two eyes with stage 2 macular holes the posterior hyaloid membrane was attached to the edge of the hole with a pseudo-operculum. In one case, the posterior hyaloid membrane was separated from the retinal surface after spontaneous closure of the macular hole, which was demonstrated in OCT images in the previously report [4]. In another eye, however, the hyaloid membrane was still attached to the edge of the hole after spontaneous closure of the macular hole. In two eyes with stage 3 macular holes, the posterior hyaloid membrane had already been detached from the macula in the initial OCT images. In the eye with a stage 4 macular hole in patient No 3, a Weiss ring was observed microscopically and the hyaloid membrane was absent in the initial OCT image. Macular hole closure was confirmed in all five eyes by OCT examination, showing sensory retina bridging over the former area of the macular hole with the appearance of foveal detachment.

The remaining 137 eyes underwent vitrectomy an average of 70 days after the initial presentation with a range between 11 and 281 days. This actual interval before surgery was a little longer than the initially scheduled averaged period of 65 days due to inconvenience in some patients.

The bridge formation of the sensory retina, which was observed in all five eyes in the present study, may be essential for spontaneous macular hole closure [5], but the detachment of posterior vitreous membrane from the fovea may not, because the left eye with stage 2 macular hole in the present study showed attachment of the hyaloid membrane after spontaneous macular hole closure. Although proliferation of glial cells on the retinal surface [6] and other unknown cellular reactions may be involved in the 
Table 1. Clinical Characteristics of the Eyes with Spontaneous Macular Hole Closure

\begin{tabular}{|c|c|c|c|c|c|c|c|c|c|c|}
\hline $\begin{array}{c}\text { Patient } \\
\text { No. }\end{array}$ & $\begin{array}{c}\text { Age } \\
\text { (Years) }\end{array}$ & Gender & R/L & $\begin{array}{c}\text { Baseline VA } \\
\text { (Decimal) }\end{array}$ & $\begin{array}{c}\text { OCT } \\
\text { Stage* }\end{array}$ & $\begin{array}{c}\text { Diameter } \\
\text { of MH }(\boldsymbol{\mu m})\end{array}$ & $\begin{array}{c}\text { Days Until } \\
\text { MH Closure }\end{array}$ & $\begin{array}{c}\text { Days Until } \\
\text { Scheduled Surgery }\end{array}$ & $\begin{array}{c}\text { Final VA } \\
\text { (Decimal) }\end{array}$ & $\begin{array}{c}\text { Days from MH } \\
\text { Closure to Final Visit }\end{array}$ \\
\hline \hline 1 & 64 & F & L & 0.8 & 3 & 150 & 57 & 89 & 0.8 & 28 \\
\hline 2 & 62 & M & L & 0.2 & 2 & 240 & 49 & 50 & 0.6 & 177 \\
\hline 3 & 71 & M & R & 0.4 & 4 & 125 & 70 & 97 & 0.8 & 441 \\
\hline 4 & 72 & M & R & 0.1 & 2 & 250 & 56 & 69 & 0.8 & 1090 \\
\hline 4 & 74 & M & L & 0.4 & 3 & 210 & 70 & 102 & 0.9 & 509 \\
\hline Mean \pm SD & $68.6 \pm 5.3$ & & & & & $195.0 \pm 55.2$ & $60.4 \pm 9.3$ & $81.4 \pm 21.6$ & & \\
\hline
\end{tabular}

$\mathrm{F}=$ female; $\mathrm{L}=$ left eye; $\mathrm{M}=$ male; $\mathrm{MH}=$ macular hole; $\mathrm{OCT}=$ optical coherence tomography; $\mathrm{R}=$ right eye; $\mathrm{SD}=$ standard deviation; $\mathrm{VA}=$ visual acuity.

*OCT stage 2, the posterior hyaloid is still attached to the hole edge in OCT image; stage 3, the posterior hyaloid is detached from the hole edge but not from the optic disk; stage 4, complete posterior vitreous detachment.

The diameter of $\mathrm{MH}$ was measured as the minimal distance between the edges of the neurosensory retina detached from the retinal pigment epithelium in the horizontal or vertical OCT image that scanned through the center of $\mathrm{MH}$.

†The duration from baseline to the time when the inner retina covered the hole was confirmed by OCT.

Patient No. 4 had the spontaneous closure of macular holes in both eyes; this patient was previously reported [4].

histological changes, the precise mechanisms of spontaneous closure of macular hole remain poorly understood.

Among several case reports on spontaneous closure of IFTMH based on OCT images alone, to our knowledge, one study reported the rate of spontaneous closure, in which 14 $(2.7 \%)$ of 510 eyes exhibited spontaneous closure [7]. The authors reported that the diameter of the closed macular hole was small between 70 to $250 \mu \mathrm{m}$ in all eyes but one. In the present study, the size of the macular hole resulting in spontaneous closure was also $250 \mu \mathrm{m}$ or smaller in all 5 eyes. They also reported that the spontaneous closure occurred prior to vitrectomy, within two months after the initial presentation, which is relatively late among clinic patients with IFTMH considering better visual prognosis in eyes with IFTMH of small size. They performed vitrectomy in the other 496 eyes, with a shorter waiting period before surgery.

Our criteria determining the scheduling of surgery was as early as possible within six months after onset without considering the macular hole size [3]. Consequently, spontaneous closure occurred in 5 eyes, 60 days after the initial presentation before the scheduled surgery 80 days after the initial presentation on average. The longer waiting period before surgery might have contributed to the higher rate of spontaneous closure of $3.5 \%$, compared to $2.7 \%$ in the previous report [7].

In conclusion small IFTMHs may close spontaneously in a small percentage of all macular hole cases. The rate of spontaneous closure may be affected by the waiting time before vitrectomy.

\section{ACKNOWLEDGEMENTS}

This study was approved by the University of Yamanashi Hospital ethics committee. This report was conducted in accordance with the Declaration of Helsinki.

\section{CONFLICT OF INTEREST}

The authors confirm that this article content has no conflicts of interest.

\section{REFERENCES}

[1] Yuzawa M, Watanabe A, Takahashi Y, Matsui M. Observation of idiopathic full-thickness macular holes. Follow-up observation. Arch Ophthalmol 1994; 112: 1051-6.

[2] Gaudric A, Haouchine B, Massin P, Paques M, Blain P, Erginay A. Macular hole formation: new data provided by optical coherence tomography. Arch Ophthalmol 1999; 117: 744-51.

[3] Bainbridge J, Herbert E, Gregor Z. Macular holes: vitreoretinal relationships and surgical approaches. Eye 2008; 22: 1301-9.

[4] Imasawa M, Chiba T, Iijima H. Spontaneous closure of idiopathic full-thickness macular holes in both eyes. Jpn J Ophthalmol 2010; 54: 507-9.

[5] Hamano R, Shimoda Y, Kishi S. Tomographic features of spontaneous closure of full-thickness macular holes. Jpn J Ophthalmol 2007; 51: 75-7.

[6] Smiddy WE, Flynn HW, Jr. Pathogenesis of macular holes and therapeutic implications. Am J Ophthalmol 2004; 137: 525-37.

[7] Privat E, Tadayoni R, Gaucher D, Haouchine B, Massin P, Gaudric A. Residual defect in the foveal photoreceptor layer detected by optical coherence tomography in eyes with spontaneously closed macular holes. Am J Ophthalmol 2007; 143: 814-9. 